P1.030 A MOLECULAR STUDY OF VAGINAL BACTERIAL COMMUNITIES AND CANDIDA ALBICANS DETECTION

doi:10.1136/sextrans-2013-051184.0251

R M Brotman, J A Schwartz, P Gajer, J Ravel, V M Bruno. University of Maryland School of Medicine, Institute for Genome Sciences, Baltimore, MD, United States

Background Vulvovaginal candidosis (VVC) is commonly diagnosed in STD clinic populations and has been found in longitudinal studies to be associated with increased risk for HIV infection. Many women with recurrent VVC relapse even when on prophylactic therapy and VVC often follows antibiotic treatment. Because the type of vaginal bacterial communities may influence yeast colonisation, we sought to compare the vaginal bacterial communities of Candida albicans-positive and C. albicans-negative women using molecular methods.

Methods Self-collected vaginal swabs were obtained cross-sectionally from 396 U.S. women equally representing four ethnic/racial groups. Exclusion criteria included self-report of vaginal discharge and pregnancy. Detection of C. albicans was performed using PCR targeting the $18 \mathrm{~S}$ rRNA gene. Vaginal bacterial composition was characterised by pyrosequencing of barcoded $16 \mathrm{~S}$ rRNA genes. The bacterial communities were clustered into five community state types (CSTs); four dominated by Lactobacillus species (L. iners, L. crispatus, L. gasseri, L. jensenii), and one lacking significant numbers of lactobacilli and characterised by higher proportions of strictly anaerobic organisms (termed CST-IV). The relationship between CSTs and C. albicans detection was evaluated by logistic regression. Random forest analysis was used to identify bacterial phylotypes associated with the presence/absence of $C$. albicans.

Results 83 women (21\%) were C.albicans-positive. Women in the L. crispatus- and L. iners-dominated CSTs were 2-fold more likely to have $C$. albicans detected compared to women in the relatively lowlactobacillus CST-IV (OR:2.42, 95\% CI: 1.21-4.85; OR:1.97, 95\% CI: 1.00-3.86, respectively). In random forest analysis, individual genera found highly associated with $C$. albicans-absence were traditional BV-associated bacteria including Campylobacter, Dialister, Peptoniphilus, Prevotella, and Anaerococcus. Lactobacillus was the primary genera associated with C. albicans-presence.

Conclusion Women with a Lactobacillus-dominated microbiota were more likely to be $C$. albicans-positive. Longitudinal studies of the vaginal microbiome are needed to determine how vaginal bacterial communities interact with Candida spp.

\section{P1.031 CD4 INDEPENDENT BINDING OF HIV TO HUMAN MANNOSE RECEPTOR ON VAGINAL EPITHELIAL CELLS AND SPERM}

doi:10.1136/sextrans-2013-051184.0252

A H Bandivdekar. National Institute for Research in Reproductive Health, Mumbai, India

Human Immunodeficiency Virus (HIV) is known to bind CD4 receptor and subsequently to CXCR4 or CCR5 coreceptors and further transmits the infection. However sperm and vaginal epithelial cells which are known to be responsible for sexual transmission of HIV are devoid of conventional CD4 receptor. HIV has been demonstrated to bind human Mannose Receptor (hMR) and enter into sperm to transmit the virus into distal cells. Subse- quent studies by immunofluorescent and Real time PCR techniques demonstrated specific binding of HIV to hMR on Vaginal epithelial cells also. Further peri-ovulatory expression of hMR on vaginal epithelial cells was found to be higher as compared to that during pre and post ovulatory period. HIV binding to hMR on vaginal epithelial cells leads to increase production of Matrix Metallo-Proteinases 9 (MMP9) which may be responsible for viral entry into the cells by inducing the cellular permeability. It was observed that less than $11 \%$ of the vaginal epithelial cells of the HIV negative females having unprotected sex with their infected sexual partners showed the presence of hMR. While 90 to $95 \%$ of vaginal epithelial cells HIV negative women from general population showed the presence of hMR. This suggests that the expression of hMR in lower number of vaginal epithelial cells protected these females from sexual transmission of HIV from their HIV infected partners. The study suggests the association of hMR in sexual transmission of HIV.

\section{P1.032 BACTERIAL SPECIES IN THE VAGINAL MICROBIOME CORRELATED BY NUGENT SCORE: CROSS-SECTIONAL DATA FROM THE MICROBICIDE SAFETY BIOMARKERS STUDY IN KENYA, RWANDA, AND SOUTH AFRICA}

doi:10.1136/sextrans-2013-051184.0253

${ }^{1}$ L Hardy, ${ }^{1} \mathrm{~V}$ Jespers, ${ }^{1} \mathrm{~J}$ Menten, ${ }^{2} \mathrm{R}$ Verhelst, ${ }^{3} \mathrm{M}$ Mwaura, ${ }^{4} \mathrm{~S}$ Delany-Moretlwe, ${ }^{5} \mathrm{G}$ Ndayisaba, ${ }^{2} \mathrm{M}$ Vaneechoutte, $6,5 \mathrm{~J}$ van de Wijgert, 'T Crucitti. 'Institute of Tropical Medicine, Antwerp, Belgium; '2University of Ghent, Ghent, Belgium; ${ }^{3}$ nternational Center for Reproductive Health, Mombasa, Kenya; ${ }^{4}$ Wits Reproductive Health and HIV Institute,

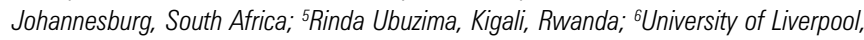
Liverpool, UK

Background Studies characterising the normal and disturbed vaginal microbiome in African women are scarce.

Methods Women were recruited in three African countries and followed up over seven visits. Vaginal specimens were examined using Nugent score defined by 0-3 (normal), a score 4-6 (intermediate) and a score of 7-10 (bacterial vaginosis (BV)) and analysed by quantitative PCR to detect and quantify lactobacilli, Gardnerella vaginalis (GV) and Atopobium vaginae (AV). The preliminary baseline data are presented.

Results Out of 376 women, $55.3 \%$ had a normal Nugent score, $7.7 \%$ an intermediate and $34.6 \%$ a BV score. A significant difference in prevalence of all species was seen between the three Nugent score groups ( $\mathrm{p}=<0.001$ except $L$. gasseri $\mathrm{p}=0.044)$. Almost all $(99.5 \%)$ women with normal Nugent score harboured lactobacilli: $L$. iners and $L$. vaginalis were frequently detected; $L$. crispatus, $L$. jensenii, $L$. gasseri were less prevalent. The percentage of women harbouring lactobacilli was lower in the intermediate group and near absent in the BV group, except for L. iners. GV and AV were detected in a high proportion of the BV group, in more than half of the women in the intermediate group and were less prevalent, but still present in the normal group.

Conclusion This is the first study to our knowledge showing a higher presence of $L$. vaginalis compared to L. crispatus mostly so in the women with normal Nugent scores. L. iners remains highly present independent of the Nugent score compared to the other lactobacilli.

Abstract P1.032 Table Frequency of detection by Nugent score

\begin{tabular}{llllllll}
\hline & L. iners & L. vaginalis & L. crispatus & L. jensenii & L. gasseri & GV & AV \\
\hline Normal $(\mathrm{n}=208)$ & $84.1 \%$ & $43.8 \%$ & $34.1 \%$ & $27.9 \%$ & $11.1 \%$ & $28.4 \%$ & $13.5 \%$ \\
Intermediate $(\mathrm{n}=29)$ & $62.1 \%$ & $31.0 \%$ & $27.6 \%$ & $17.2 \%$ & $13.8 \%$ & $69.0 \%$ & $58.6 \%$ \\
BV $(\mathrm{n}=130)$ & $66.0 \%$ & $6.9 \%$ & $6.2 \%$ & $3.8 \%$ & $3.8 \%$ & $93.8 \%$ & $82.3 \%$ \\
\hline
\end{tabular}

